\title{
Extraction of Equivalent Circuit Parameter of the GIS Pipe Spacer
}

\author{
Yonghong Wang ${ }^{1}$, Bing Xiao ${ }^{1}$, Guizhong Qiu ${ }^{1}$, Lipeng Bi ${ }^{1}$, Keqiang $\mathrm{Xu}^{2}$, Chongqing Jiao ${ }^{3}$ and Shuyun $\mathrm{Cao}^{3^{*}}$ \\ ${ }^{1}$ Power Science Research Institute, State Grid Inner Mongolia East Electric Power co., LTD, Hohhot, 010090, China \\ ${ }^{2}$ Hengjingtong Electronic Technology Co., Ltd, Nanjing, 210018, China \\ ${ }^{3}$ School of Electrical Engineering, North China Electric Power University, Beijing, 102206, China
}

\begin{abstract}
In Gas Insulated Switchgear (GIS) substation, the spacer used for supporting conductor, isolating air chamber and insulation is a very important insulation component in GIS pipe, and it is important to study the spacer to ensure the insulation performance and quality of GIS. Local distortion of electric field distribution will be produced when electromagnetic wave propagates to spacers. In this article, the static capacitance calculated by electromagnetic simulation is used to model spacers.
\end{abstract}

\section{Introduction}

With high amplitude and broadband electromagnetic waves caused by switching operation propagating in gas insulated switchgear (GIS) pipe, vary fast transient over voltage (VFTO) will be produced in GIS [1-3]. Field distribution of electromagnetic wave propagating in GIS can be distorted locally at the discontinuous/abrupt positions like spacers. Setting up equivalent circuit model of basin-type insulator correctly is very important to study the VFTO [4-8].

Spacers cast by epoxy resin under high vacuum are widely used in GIS pipe. The electric field distribution will be changed around spacers [9], and the electromagnetic wave is no longer TEM or quasi TEM [10]. Ansoft Maxwell is a kind of electromagnetic field simulation software based on finite element method, and the static capacitance calculated by electromagnetic simulation is used to replace the transmission line mode to simulate the spacer. Then, a simplified capacitance calculation method of spacers is proposed in the paper.

\section{Principle of extracting static capacitance by finite element software}

Ansoft Maxwell based on finite element method, has an intuitive and easy-to-use graphical user interface (GUI) and an automatic adaptive mesh solver, and it can not only display the electromagnetic field distribution of the object, but also automatically calculate the electromagnetic force, inductance and capacitance.

The calculation of static capacitance is mainly through the calculation of electric field energy by software of Ansoft Maxwell, the formula of the electric field energy stored between two conductors due to capacitive coupling as follows in formula (1) for passive field [11].

$$
W_{i j}=\frac{1}{2} \int_{\Omega} D_{i} \cdot E_{j} d \Omega
$$

Where, electric field energy between conductor $i$ and $j$ is represented by $W_{i j}$, electric displacement vector caused by conductor $i$ and electric field intensity caused by conductor $j$ represented by $D_{i}$ and $E_{j}$. The capacitance between the two conductors can be obtained as formula (2) when the potential distribution is known in the calculation area.

$$
C_{i j}=\frac{2 W_{i j}}{\left(V_{i}-V_{j}\right)^{2}}
$$

\section{Calculation of distortion capacitance of spacers}

Capacitance of the coaxial structure of transmission line per unit length is as follows:

$$
C_{0}=\frac{2 \pi \varepsilon}{\ln \left(r_{s} / r_{w}\right)}
$$

Where, dielectric constant between coaxial conductors is represented by $\varepsilon$, meanwhile, $r_{w}$ and $r_{s}$ are radius of inner and outer conductor respectively.

For $1100 \mathrm{kV}$ UHV GIS pipe, the relative dielectric constant of spacers and $\mathrm{SF}_{6}$ are 5.2 and 1.002, and the

*Corresponding author's e-mail: 546645167@qq.com 
radius of inner and outer conductor of pipe are $90 \mathrm{~mm}$ and $440 \mathrm{~mm}$. Capacitance of the pipe per unit length $C_{01}$ can be calculated when insulating material is $\mathrm{SF}_{6}$ only in

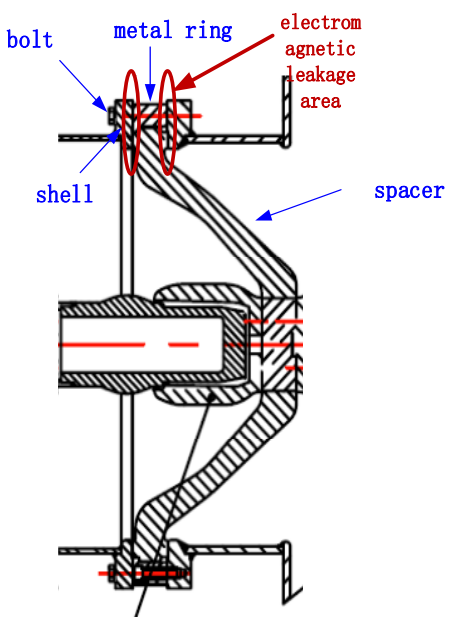

(a) Plane structure. pipe, where $C_{01}=35.108 \mathrm{pF} / \mathrm{m}$. The plane structure and three-dimensional diagram of the basin insulator are shown in Fig.1

Figure 1. Structure diagram of spacers.

The 2D module is used to simulate spacers because of its symmetrical structure, and its electric field distribution is shown in Figure 2(a) by simulation when the length of pipe is $1.2 \mathrm{~m}$.

From the Figure 2(a) we can see that the area of electric field distortion is within the range and the value of capacitance $C$ is $50.803 \mathrm{pF}$, and the equivalent circuit model of spacer is shown in Figure 2(b), then the compensation capacitance in the electric field distortion region is:

$$
\Delta C=C-l \times C_{01}
$$

Bring in the data and get the static compensation capacitance is $8.6734 \mathrm{pF}$.

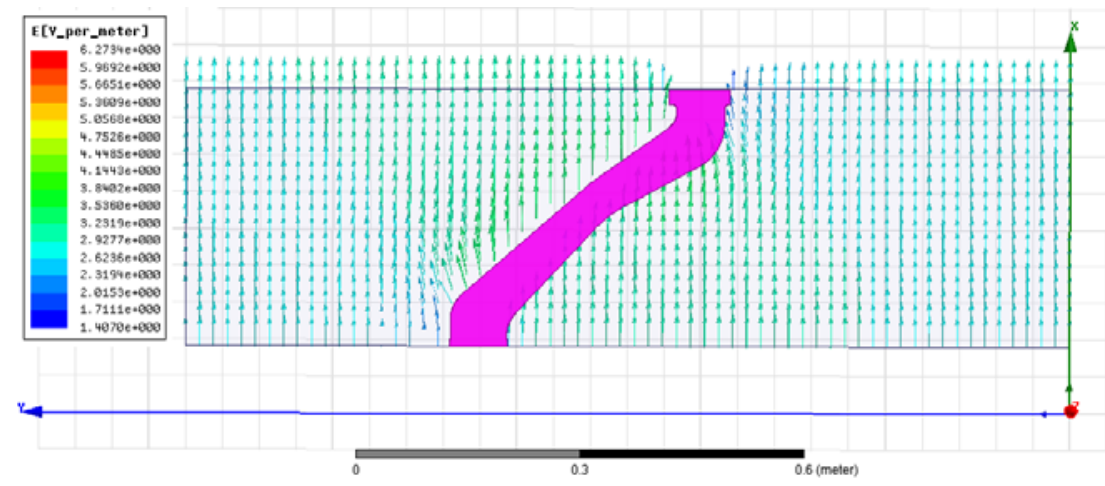

(a) Electric field distribution of spaces

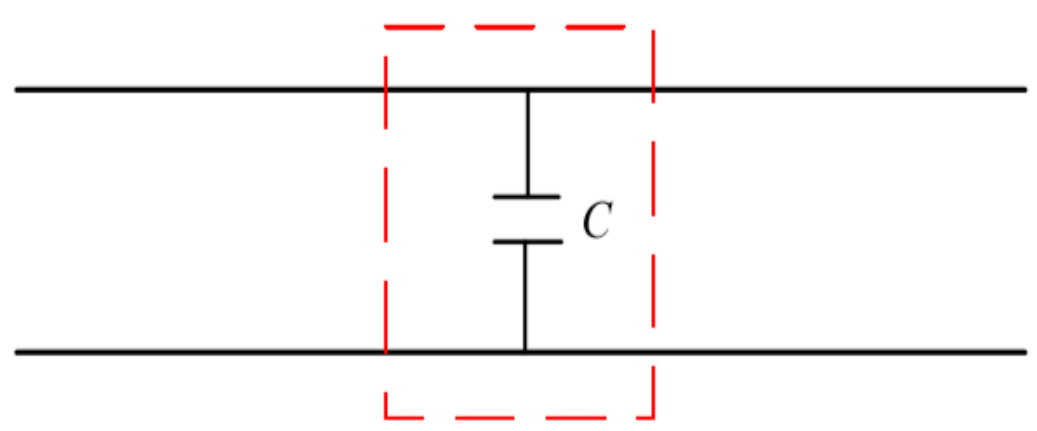

(b) Equivalent circuit model of spacer

Figure 2. Electric field distribution of spaces 


\section{Accuracy verification of Ansoft Maxwell}

Comparing the simulation results of two kinds of software of Ansoft Maxwell and ANSYS used to verify the correctness of Ansoft Maxwell under the same conditions.

Simulation model of spacer in figure 3 , consisted by parallelograms of equal area and different angles, have the same base length $l$, and angle between spacers and central conductor is represented by $\theta$, then the thickness of spacer is $d=l \times \sin \theta$.

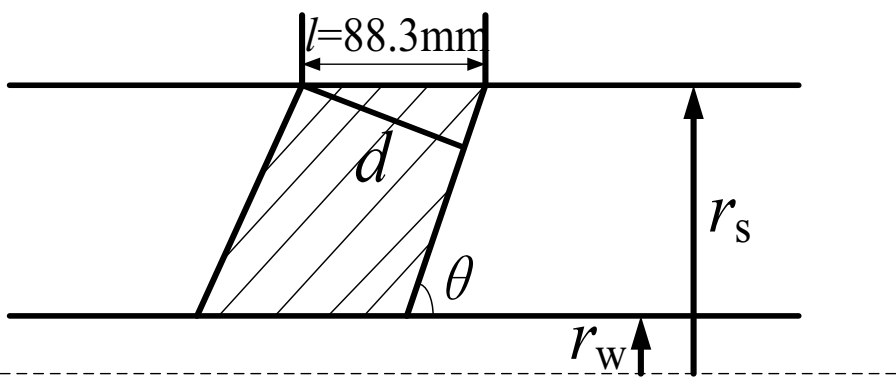

Figure 3. Simulation model of spacer with angle variation

For the simulation model in figure 3 , when the voltage of inner conductor is $1 \mathrm{~V}$ and that of outer conductor is $0 \mathrm{~V}$, and values of $\theta$ are $30^{\circ}, 45^{\circ}, 60^{\circ}$, $75^{\circ}$ and $90^{\circ}$ respectively, the value of total capacitance of spacer calculated by two kinds of software and the relative error of two kinds of software is shown in table 1 .

Table1. Comparison of total capacitance value of spacer

\begin{tabular}{cccl}
\multicolumn{4}{c}{ between ANSYS and Maxwell } \\
\hline$\theta\left(^{\circ}\right)$ & $\begin{array}{c}\text { ANSYS } \\
(\mathrm{pF})\end{array}$ & $\begin{array}{c}\text { Maxwell } \\
(\mathrm{pF})\end{array}$ & $\begin{array}{l}\text { Relative } \\
\text { error } \%\end{array}$ \\
\cline { 2 - 4 } 90 & 55.145 & 55.146 & 0.0018134 \\
75 & 54.601 & 54.595 & 0.01099 \\
60 & 53.02 & 53.016 & 0.0075449 \\
45 & 50.615 & 50.607 & 0.0158081 \\
30 & 47.872 & 47.865 & 0.0146245 \\
\hline
\end{tabular}

With the change of spacer angle, the maximum relative error is not more than $0.02 \%$ between two kinds of software, and the result of static capacitance can be calculated exactly by Maxwell.

\section{Analysis of compensation capacitance of spacers}

The effect of electric distortion at the discontinuous/abrupt positions like spacers is usually regarded as a lumped circuit element inserted into the transmission line model, such as compensation capacitance [12]. With the change of spacer structure, the compensation capacitance of electric field distortion of spacer will also change.

Simulation model of rectangular spacer with the same thickness as spacer model in figure 3 is shown in figure 4 , and cross-sectional area is unchanged when $\theta$ are taken as $5^{\circ}, 10^{\circ}, 15^{\circ}, 20^{\circ} \ldots$ and $90^{\circ}$ in figure 3 . For $1100 \mathrm{kV}$ GIS pipe and $220 \mathrm{kV}$ GIS pipe, compensation capacitance values of the two simulation models in figure 3 and figure 4 are used for comparison and analysis with the change of $\theta$.

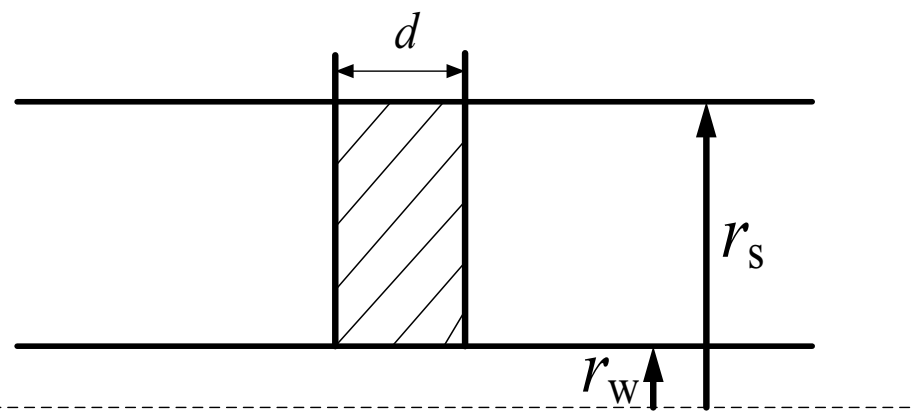

Figure 4. Simulation model of rectangular spacer

\subsection{Analysis of compensation capacitance of $1100 k V$ GIS spacer}

For $1100 \mathrm{kV}$ GIS pipe, the relative dielectric constant of spacers and $\mathrm{SF}_{6}$ are 5.2 and 1.002, and the radius of inner and outer conductor of pipe are $90 \mathrm{~mm}$ and $440 \mathrm{~mm}$. According to simulation models in figure 3 and figure 4, then results of compensation capacitance are shown in Figure 5. 


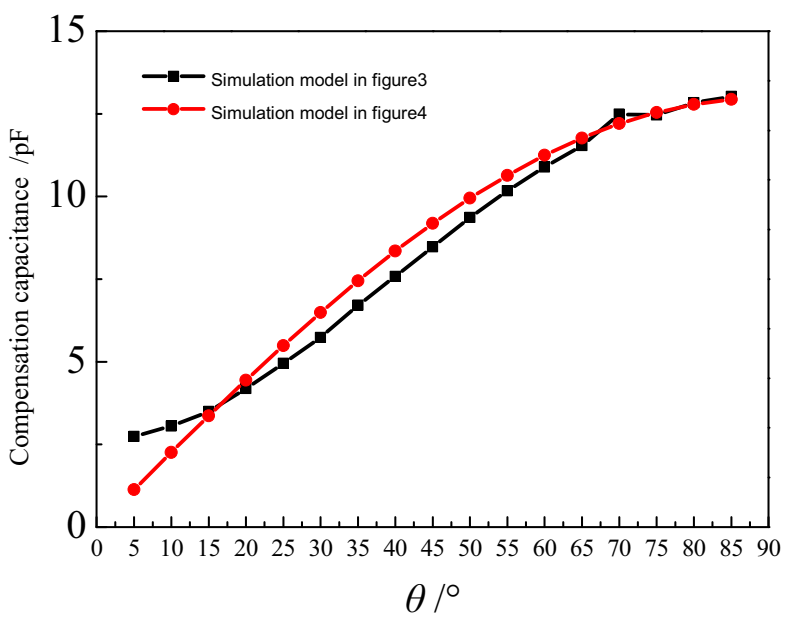

Figure 5. Compensation capacitance of $1100 \mathrm{kV}$ GIS spacer

The compensation capacitance of the spacer increases with the increase of the angle in Figure 5, and the maximum relative error of the value of compensation capacitance between two simulation models is about $10 \%$ at the same angle.

\subsection{Analysis of compensation capacitance of 220kV GIS spacer}

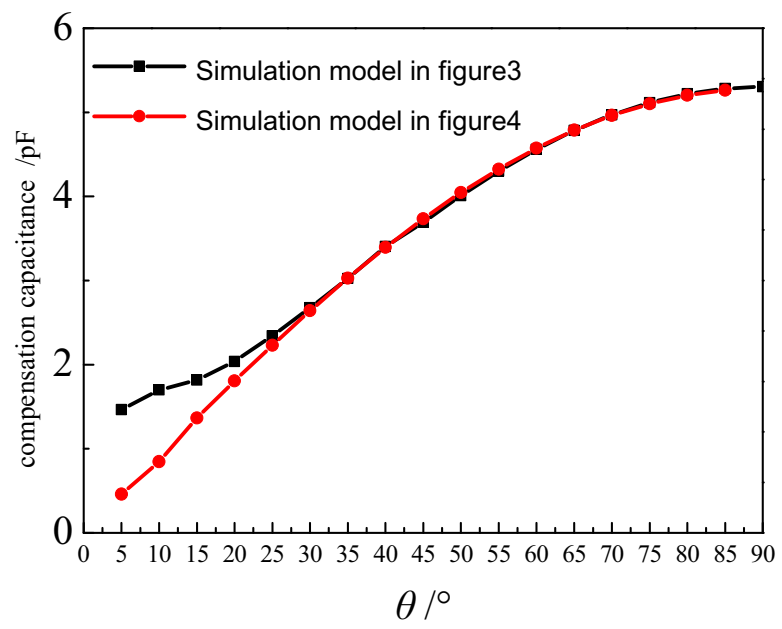

Figure 6. Compensation capacitance of $220 \mathrm{kV}$ GIS spacer

With the increase of the angle, the compensation capacitance value increases, and the compensation capacitance value in two cases basically coincides from the Figure 6.

\section{6 conclusions}

The static capacitance calculated by electromagnetic simulation can be used to model spacers, and for $1100 \mathrm{kV}$ GIS pipe, the static compensation capacitance is $8.6734 \mathrm{pF}$. The compensation capacitance of the same area spacer increases with the increase of the angle, and the compensation capacitance of the spacer with a certain
For $220 \mathrm{kV}$ GIS pipe, the relative dielectric constant of spacers and $\mathrm{SF}_{6}$ are 4.0 and 1.002, and the radius of inner and outer conductor of pipe are $30 \mathrm{~mm}$ and $320 \mathrm{~mm}$. According to simulation models in figure 3 and figure 4, where $l=75 \mathrm{~mm}$, then results of compensation capacitance are shown in Figure 6.

$$
\theta / 0
$$

angle can be approximately equivalent to that of the rectangular insulator with the same thickness, which can simplify the calculation of the compensation capacitance of the spacer.

\section{Acknowledgments}

This project was supported by the State Grid Inner Mongolia East Electric Power co., ltd. Power Science Research Institute. 


\section{References}

1. Huamao Zhan, Chengnan Zhao, Shaofeng Duan etc. (2013) Measuring of Very Fast Transient Over-voltage and Very Fast Transient Current Generated by Disconnector in Gas Insulated Switchgear Switching on/off Short Buses with no Load. High Voltage Engineering, 39(6): 13171323.

2. Xiaojian Li, Yao Zhong, Ren Wang, Hong Huo. (2012) Time-frequency Domain Characteristics of Electromagnetic Disturbance in Extra High Voltage System. High Voltage Engineering, 38(11):2889-2898.

3. Rao M M, Thomas M J, Singh B P. (2007) Transients induced on control cables and secondary circuit of instrument transformers in a GIS during switching operations[J]. IEEE Transactions on Power De-livery, 22(3): 15051513.

4. Hengtian Wu, Chongqing Jiao, Xiang Cui. (2018) Study on Coupling of Very Fast Transients to Secondary Cable via a Test Platform. IEEE TRANSACTIONS ON ELECTROMAGNETIC COMPATIBILITY, VOL. 60, NO. 5.

5. Hengtian $\mathrm{Wu}$, Xiang Cui, Xiaofan Liu, Chongqing Jiao, Rong Hu, Weijiang Chen, Lei Wang. (2017) Characteristics of Electromagnetic Disturbance for Intelligent Component Due to Switching Operations via a $1100 \mathrm{kV}$ AC GIS Test Circuit. IEEE TRANSACTIONS ON POWER DELIVERY, VOL. 32, NO. 5

6. Weidong Zhang, Peilong Chen, Weijiang Chen etc. (2013) Measurement and Simulation of
Disturbance Voltage Generated by VFTO in UHV GIS Substation on the Secondary Cables. Proceedings of the CSEE, 33(16):187-196+2.

7. Okabe S., Takami J., Nojima K. (2008) Circuit models of gas insulated switchgear elements for electromagnetic wave leakage analysis. IEEE Transactions on Dielectrics and Electrical Insulation, 15(5): 1439-1448.

8. Z.Haznadar, S.Carsimamovic, R.Mahmutcehajic. (1991) More accurate modeling of gas insulated substation components in digital simulations of very fast electromagnetic transients. Power Industry Computer Application Conference, 260267, DOI: 10.1109/PICA.

9. Binxian Lu, Zhun Meng. (2013) The Research of Gas Insulated Switchgear Model Simplification and Its Influence on Simulation Result Based on Manufacturing Feature. Transactions of China Electrotechnical Society, 28(1):119-125.

10. Shuhei K,Shigemitsu O. (2009) Electromagnetic Wave Radiated from an Insulating Spacer in Gas Insulated Switchgear with Partial Discharge Detection. IEEE Transactions on Dielectrics and Electrical Insulation.

11. Mingyang Li. (2016) Broadband Equivalent Circuit Model of Bushing for Gas Insulated Switchgear in UHV Substation.

12. Chongqing Jiao, Mingyang Li. (2015) Calculation and Analysis of Compensation Capacitance Caused by Radius Discontinuities in Coaxial Structure. Transactions of China Electrotechnical Society, 30(S2):1-6. 